Aims. Measure compliance with National Institute for Health and Care Excellence (NICE) recommendations in four Adult CMHT's Guide further service development.

We audited the case notes of 20 patients each currently under care of 9 General Adult Consultants across 6 CMHT's in East side of North Wales against NICE standards using an adapted version of the ADHD audit support tool.

My role in the Project \& How does this represent my practice? I was the audit and overall lead for this project

I formulated the audit tool and registered my project with Audit Registration Team.

I lead data collection and compilation of results.

Method. Overall, this is the first audit of Adult ADHD Services in East side of North Wales.

It established good compliance with NICE guidance for assessment and treatment.

NICE has expressed the need for full mental health and social assessment including full history and physical examination prior to the drug treatment.

Good compliance was observed in using \& documenting Diagnostic Criteria (DSM-IV and/or ICD-10).

There were deficiencies in conducting or arranging recommended physical examination \& side effect monitoring.

Drug treatment was the first line of treatment in the majority of cases.

Antipsychotics were used in some patients referred for ADHD assessment, despite the fact that NICE has ruled out the use of antipsychotic drugs in treatment of core symptoms of ADHD.

Result. Prevalence of Adult ADHD clinician case load in Wrexham and Fintshire Counties.

Diagnosis of Adult ADHD according to ICD 10 \& DSM IV Guidelines.

Pre treatment screening of physical health for ADHD patients.

Side effects monitoring of patients on stimulant medications.

Conclusion. The finding highlights the need for more effort in educating clinicians about safety and effectiveness of antipsychotics in ADHD.

Comprehensive treatment programmes that address psychological, behavioural, educational and occupational needs should be established.

Development of local ADHD Clinics, support groups and in partnership with the voluntary sector should be encouraged.

It is important that mental health professionals receive appropriate training in assessment, management \& monitoring of ADHD patients with co morbid substance use disorder and other mental illnesses.

BETSI Health Board to participate in national Prescribing Observatory for Mental Health (POMH-UK) Quality Improvement Programme (QIP) focusing on prescribing for ADHD in children, adolescents \& adults.

\section{Clozapine clinics in north Wales - service evaluation audit}

Jawad Raja ${ }^{1 \star}$, Zeenish Azhar ${ }^{2}$ and Masood Malik ${ }^{3}$

${ }^{1}$ ST5 Betsi Cadwaladr University Health Board; ${ }^{2}$ CT2 Betsi Cadwaladr University Health Board and ${ }^{3}$ Clinical Director Betsi Cadwaladr University Health Board

${ }^{\star}$ Corresponding author.

doi: 10.1192/bjo.2021.900

\section{Aims.}

1. Quality of clozapine clinic appointment

2. Effectiveness of clozapine clinic servive
3. Compliance with BCUHB guidelines for physical health monitoring in clozapine clinics

4. We retrospectively audited 40 case notes 10 each from 4 differtent CMHT clozapine clinics

My role in the Project \& How does this represent my practice?

1. I was the audit and overall lead for this project

2. I formulated the audit tool and registered my project with Audit Registration Team

3. I lead data collection and compilation of results

\section{Background.}

1. This audit followed up from a Coroner's investigation for a clozapine clinic patient

2. Clozapine is used for Treatment Resistant Schizophrenia but needs close monitoring due to potentially fatal side effects

3. NICE recommends annual monitoring of weight, blood pressure, waist measurement, blood glucose and plasma lipid levels

\section{Method.}

1. Has the patient been seen in the past year by clinician to monitor response to clozapine treatment?

2. Has the clozapine plasma level been measured during the last year of treatment?

3. Is brief MSE \& Risk assessment documented during review?

4. Has Life style modification advice been provided?

5. Has annual physical health been completed?

6. Has Annual CTP/CPA been completed and documented?

7. Has the patient been allocated a named care coordinator?

8. Has clozapine side effects monitoring been documented?

\section{Conclusion.}

1. Clozapine is a superior medication for the treatment of refractory schizophrenia and is also be effective for other conditions

2. Clozapine is underused due to a variety of barriers related to the drug and its properties, the health care system \& regulatory requirements

3. This service evaluation/quality improvement project provides the framework for clozapine clinics evaluation and recommends strategies for improvement

Service evaluation of primary care mental health support services in north Wales

Jawad Raja ${ }^{1 \star}$, Alberto Salmoiraghi ${ }^{2}$ and Zeenish Azhar $^{3}$

${ }^{1}$ ST5 Betsi Cadwaladr University Health Board; ${ }^{2}$ Medical Director Betsi Cadwaladr University Health Board and ${ }^{3}$ CT3 Trainee Betsi Cadwaladr University Health Board

${ }^{\star}$ Corresponding author.

doi: 10.1192/bjo.2021.901

Aims. Bringing specialist psychiatrist into PCMHT Undertaking initial assessments for people Referred by G.P's Working According to the principle of "Prescribing Interventions"

Decrease number of assessments carried out within secondary Care

Method. County of Wrexham is situated between the lower Dee Valley and the Welsh mountains. It is the largest town in North Wales $(140,000)$

Since 2013, the total new patient referrals to be seen by Wrexham county consultant psychiatrists has consistently risen 